\section{POS0106 BILAG-2004 LDA AND BST LDA ARE VALID TREAT TO TARGET IN SLE}

C. S. Yee ${ }^{1}$, C. Gordon ${ }^{2}$, M. Akil ${ }^{3}$, P. Lanyon ${ }^{4}$, C. J. Edwards ${ }^{5}$, D. Isenberg ${ }^{6}$, A. Rahman ${ }^{6}$, L. S. Teh ${ }^{7}$, S. Tosounidou ${ }^{8}$, R. Stevens ${ }^{1}$, A. Prabu' ${ }^{2}$, B. Griffiths ${ }^{9}$, N. Mchugh ${ }^{10}$, I. N. Bruce ${ }^{11}$, Y. Ahmad ${ }^{12}$, M. Khamashta ${ }^{13}$, V. Farewell ${ }^{14}$ on behalf of British Isle Lupus Assessment Group (BILAG). ${ }^{1}$ Doncaster \& Bassetlaw Teaching Hospitals NHS Foundation Trust, Department of Rheumatology, Doncaster, United Kingdom; ${ }^{2}$ University of Birmingham, Rheumatology Research Group, Birmingham, United Kingdom; ${ }^{3}$ Sheffield Teaching Hospitals NHS Foundation Trust, Department of Rheumatology, Sheffield, United Kingdom; ${ }^{4}$ Nottingham University Hospitals NHS Trust, Department of Rheumatology, Nottingham, United Kingdom; ${ }^{5}$ University Hospital Southampton NHS Foundation Trust, Department of Rheumatology, Southampton, United Kingdom; ${ }^{6}$ University College London, Centre for Rheumatology Research, London, United Kingdom; ${ }^{7}$ Royal Blackburn Hospital, Department of Rheumatology, Blackburn, United Kingdom; ${ }^{8}$ Sandwell and West Birmingham Hospitals NHS Trust, Department of Rheumatology, Birmingham, United Kingdom; ${ }^{9}$ Newcastle upon Tyne Hospitals NHS Foundation Trust, Department of Rheumatology, Newcastle, United Kingdom; ${ }^{10}$ Royal National Hospital for Rheumatic Diseases NHS Trust, Department of Rheumatology, Bath, United Kingdom; ${ }^{11}$ The University of Manchester, Centre for Epidemiology Versus Arthritis, Manchester, United Kingdom; ${ }^{12}$ Betsi Cadwaldr University Health Board, Department of Rheumatology, Wales, United Kingdom; ${ }^{13}$ Guy's and St Thomas' NHS Foundation Trust, Department of Rheumatology, London, United Kingdom; ${ }^{14}$ University of Cambridge, MRC Biostatistics Unit, Cambridge, United Kingdom

Background: Low disease activity state has been defined using SLEDAI and used as treatment target in SLE. However, there has not been any such definition using BILAG-2004 index (BILAG-2004).

Objectives: This study was to determine if low disease activity state according to BILAG-2004 is valid for use as treatment target in SLE. We also assessed disease activity longitudinally using BILAG-2004 systems tally (BST). BST is an alternative way of representing BILAG-2004 scores that combines the flexibility and simplification of numerical scoring of BILAG-2004 with the clinical intuitiveness of BILAG-2004 structure.

Methods: This was a prospective multi-centre longitudinal study in the UK of an inception cohort of SLE patients (recruited within 12 months of achieving 1997 ACR revised criteria for SLE). Data were collected on disease activity (BILAG-2004 and BILAG2004-Pregnancy Index during pregnancy), SLICC/ACR DI (SDI), cumulative drug exposure and death at every visit. This study ran from 1st January 2005 to 31st December 2017. Four low disease activity states (LDA) were defined using BILAG-2004: 1) BILAG-2004 LDA when all 9 systems had scores of C, D or E on assessment (no Grade A or B), 2) BST LDA when there was persistent score of $C$, D or E in all 9 systems between 2 consecutive visits (equivalent to 2 consecutive visits with BILAG-2004 LDA), 3) BILAG-2004 Remission when all 9 systems had scores of $D$ or $E$ on assessment and 4) Persistent Remission when there was persistent score of $D$ or $E$ in all 9 systems between 2 consecutive visits. Longitudinal analysis using Poisson regression with random effects model was used with development of new damage as the outcome of interest. Gender, cardiovascular risk factors, antiphospholipid syndrome status and most drugs (except hydroxychloroquine, glucocorticoids, mycophenolate and cyclophosphamide) were excluded from the model as they were not associated with development of damage in univariate analysis.

Results: 273 patients were recruited (91.2\% female, 59.3\% Caucasian, $17.2 \%$ African/Caribbean, $17.2 \%$ South Asian) with mean age at recruitment of 38.5 years (SD 14.8). $97.8 \%$ had no damage at recruitment $(2.2 \%$ had SDI score of 1). Median follow-up was 73.4 months (range: $1.8,153.8$ ) with total follow-up of 1767 patient-years. There were 13 deaths and 114 new damage items occurred during follow-up. There were 6674 assessments with disease activity score: 319 assessments with Grade A activity in 95 patients (84.6\% had only 1 system with grade A, range: 1 - 4) and 1704 assessments with Grade A or B activity in 239 patients $(78.7 \%$ had only 1 system with Grade A or B, range: 1 - 5).

BILAG-2004 LDA was achieved in $74.5 \%$ of assessments (from 271 patients). BILAG-2004 Remission occurred in $28.2 \%$ of assessments (from 234 patients). 6401 observations with BST were available (1 observation derived from change in activity between 2 consecutive assessments) and $63.7 \%$ were in BST LDA. There was no observation with Persistent Remission between consecutive visits. Table 1 summarises multivariate analysis which showed BILAG-2004 LDA to be inversely associated with damage. Similar results were obtained with BILAG2004 Remission (RR 0.60 with $95 \% \mathrm{Cl} 0.38,0.96$ ) and BST LDA (RR 0.65 with $95 \% \mathrm{Cl} 0.43,0.99)$. Cumulative drug exposure since recruitment for mycophenolate was protective against new damage (RR 0.99 with $95 \% \mathrm{Cl} 0.99,0.99$ ).
Table 1.

Variable Relative Risk $(95 \% \mathrm{Cl})$ for New Damage

Ethnicity

Afro-Caribbean

South Asian

Others

Age at diagnosis

Prior SDI score

BILAG-2004 LDA

Hydroxychloroquine since last visit (per g)

Steroids since last visit (per $100 \mathrm{mg}$ )

Cyclophosphamide since last visit (per g)

$1.22(0.68,2.18)$

$1.81(0.97,3.38)$

$2.22(0.63,7.85)$

$1.06(1.04,1.08)$

$0.68(0.43,1.06)$

$0.60(0.39,0.94)$

$0.99(0.98,0.99)$

$1.02(1.01,1.03)$

$1.67(1.15,2.41)$

Conclusion: BILAG-2004 LDA and BST LDA are valid treatment targets in SLE. BILAG-2004 Remission and Persistent Remission are uncommon, which make them unrealistic as a treatment target.

REFERENCES:

[1] Yee C. S., et al. The BILAG-2004 systems tally - a novel way of representing the BILAG-2004 index scores longitudinally. Rheumatology (Oxford) 2012; 51[11]: 2099-2105.

Acknowledgements : Versus Arthritis, Vifor Pharma

Disclosure of Interests: Chee-Seng Yee Consultant of: Bristol Myers Squibb, ImmuPharma, Grant/research support from: Vifor Pharma, Caroline Gordon Speakers bureau: UCB, Consultant of: Center for Disease Control, Astra-Zeneca, MGP, Sanofi and UCB, Mohammed Akil: None declared, Peter Lanyon: None declared, Christopher John Edwards Consultant of: Glaxo Smith Kline, Roche, Grant/research support from: Glaxo Smith Kline, Roche, David Isenberg: None declared, Anisur Rahman: None declared, Lee-Suan Teh: None declared, Sofia Tosounidou: None declared, Robert Stevens: None declared, Ahtiveer Prabu: None declared, Bridget Griffiths: None declared, Neil McHugh: None declared, Ian N. Bruce: None declared, Yasmeen Ahmad: None declared, Munther Khamashta: None declared, Vernon Farewell: None declared

DOI: 10.1136/annrheumdis-2021-eular.10

\section{POS0107 TAPERING BELIMUMAB IN PATIENTS WITH SLE: A SINGLE CENTER EXPERIENCE}

M. Fredi ${ }^{1}$, T. Vojinovic ${ }^{2}$, F. Regola ${ }^{1}$, G. Babaglioni ${ }^{3}$, D. Bettoni ${ }^{3}$, I. Cavazzana ${ }^{2}$, F. Franceschini ${ }^{1} .{ }^{1}$ UO Reumatologia e Immunologia Clinica ASST Spedali Civili e Università degli Studi di Brescia, ASST Spedali Civili, Brescia, Italy; ${ }^{2}$ UO Reumatologia e Immunologia Clinica, ASST Spedali Civili, Brescia, Italy; ${ }^{3}$ Farmacia Aziendale, ASST Spedali Civili, Brescia, Italy

Background: In Italy, the use of belimumab has been authorized since 2013, in patients with active systemic lupus erythematosus (SLE) with positive anti-double-stranded DNA and low C3 or C4 levels, despite standard therapy. Belimumab is effective in reducing disease activity and number of flares and in blocking damage progression. To our knowledge, no data are available on dose tapering of belimumab in SLE patients chronically treated with it.

Objectives: The aim of this retrospective study was to analyse prevalence of tapering in a single cohort and to evaluate disease activity after tapering.

Methods: Patients, who received intravenous belimumab $(10 \mathrm{mg} / \mathrm{kg})$ for at least 12 months between June 2013 and December 2020 were enrolled. The Systemic Lupus Erythematosus Disease Activity Index 2000 (SLEDAI-2K), the Systemic Lupus International Collaborating Clinics/American College of Rheumatology Damage Index (SLICC-SDI), Physician Global Assessment (PGA), C3 and C4 levels, and prednisone daily dose were recorded at baseline and every sixmonths. Follow-up was stopped when patients switched to subcutaneous belimumab or when it was discontinued for more than 6 months (interrupted). Dose tapering was defined as the prolongation of the regular dose interval, after initial treatment based on the registered dosage.

Results: Between June 2013 and December 2020, 48 patients received intravenous belimumab for at least 12 months (100\% Caucasians, $94 \%$ females). All patients received standard belimumab dosage of $10 \mathrm{mg} / \mathrm{Kg}$ every 4 weeks. Fourteen $(29.1 \%)$ of 48 patients lengthened the dosing interval to 5 weeks; a subsequent lengthening of the interval occurred in 7 patients every 6 weeks and in 1 patient belimumab was gradually tapered to every 13 weeks.

Twelve months (T12) after belimumab introduction, 3 patients $(6.2 \%)$ were receiving a tapered dosage; at T24, 9 out of the $37(24.3 \%)$, at T36 9 out of 21 (42.8\%), and at T48 8 out of 18 (44.4\%). Differences in disease activity, damage, concomitant medications, disease duration at belimumab introduction (baseline) were evaluated among patients that maintained the standard interval versus the group that received a tapered dose. Notably, no differences were found among the two groups (standard dose vs tapering) concerning median age at baseline (41, IQR 31-47.5 vs 38, IQR 32.2-51.5), disease duration (median 11, IQR 7.2-16 